\title{
Campo de Flujo no Estacionario en un Modelo del Múltiple de Admisión de un Motor de Combustión Interna
}

\author{
Dámazo N. Jiménez ${ }^{(1)}$ y Francisco J. Solorio(2) \\ (1) Instituto Tecnológico de Apizaco, Av. Instituto Tecnológico s/n, \\ 90300 Apizaco, Tlax.-México (e-mail: xadanistine@hotmail.com) \\ (2) Departamento de Termoenergía, Facultad de Ingeniería, UNAM, \\ Ciudad Universitaria, 04510, México, D.F.-México (e-mail: fjso@servidor.unam.mx)
}

\begin{abstract}
Resumen
Se obtiene y analiza el campo de flujo no estacionario que se genera en un modelo simplificado del múltiple de admisión de un motor de combustión interna, que consta de una cámara de distribución y cuatro derivaciones. El modelo se instala en la succión de un motor sin combustión, arrastrado mediante un motor eléctrico. Las velocidades se miden con un anemómetro láser Doppler y se promedian en fase en 25 ciclos del motor, presentándose como función del ángulo de giro del cigüeñal para algunos puntos de interés. Además, se muestra el campo de flujo completo para ciertos ángulos de giro seleccionados, señalándose la evolución de los vórtices de entrada a las derivaciones y las zonas de recirculación en las esquinas de la cámara de distribución.
\end{abstract}

Palabras clave: múltiple de admisión, campo de flujo, anemometría láser Doppler, combustión

\section{Unsteady Flow Field in a Model of the Intake Manifold of an Internal Combustion Engine}

\begin{abstract}
The unsteady flow field generated in a simplified model of the intake manifold of an internal combustion engine containing a plenum and four runners is proposed and analyzed. The model is mounted in the intake system of an engine under motored conditions, driven by an electric motor. Instantaneous ensemble average velocities are obtained for 25 engine cycles and reported as a function of the crank angle for some representative points. Also, the global flow field is presented for selected crank angles, showing the evolution of the unsteady eddies produced near the runner inlet and the secondary flows generated in the plenum corners.
\end{abstract}

Keywords: intake manifold, flow field, laser Doppler anemometry, combustion 


\section{INTRODUCCIÓN}

La geometría del múltiple de admisión es de gran importancia en los motores de combustión interna modernos debido a su influencia directa en la eficiencia volumétrica y en las características del flujo de entrada al cilindro, lo que repercute en el desempeño integral del motor, sobre todo en lo relacionado con su potencia, consumo de combustible y emisiones contaminantes.

El flujo en el sistema de admisión es no permanente por la naturaleza misma de los procesos del motor. Además, en general es turbulento y presenta variaciones cíclicas de alta frecuencia, lo que complica su estudio teórico y hace que en el terreno experimental se tengan que usar técnicas de medición que, además de obstruir lo menos posible al flujo, muestren una buena resolución espacial y temporal. En este sentido Yeoman y Taylor (1978) demuestran la capacidad de aplicación de la anemometría láser Doppler (ALD) en el estudio de estos flujos, al obtener los perfiles de velocidades medias y sus rms en algunas secciones del múltiple de admisión de un motor a gasolina de 4 cilindros, bajo condiciones de motor arrastrado. Identificaron la dependencia de la velocidad con el ángulo de giro del cigüeñal, pero debido al número limitado de secciones donde midieron no pudieron detectar ninguna estructura espacial del flujo.

Por otro lado, Arcoumanis y Flamang (1989) estudian el flujo transitorio en el múltiple de admisión de un motor diesel de línea que opera arrastrado, también de 4 cilindros, pero en el que sólo uno se mantiene en compresión y los tres restantes se encuentran abiertos a la atmósfera. Usan ALD para medir la velocidad promediada en fase y sus rms en varios puntos de tres secciones, dos de ellas localizadas en corredores adyacentes, y la tercera en la cámara de distribución o plenum, cerca de la unión con los corredores en estudio. Concluyen que el flujo en los corredores es prácticamente unidimensional y repetitivo de ciclo a ciclo, con niveles bajos de turbulencia. En el plenum se presenta un flujo muy complejo como resultado de la interacción del proceso de admisión en los diferentes cilindros.

Para estudiar los aspectos fundamentales de los flujos en los múltiples, sin los inconvenientes que presentan las complejas geome- trías de los múltiples de línea, varios autores han usado modelos con formas simplificadas. A continuación se mencionan algunos trabajos importantes en este sentido.

Durst el al. $(1989,1992)$ presentan un trabajo experimental sobre el campo de flujo en un tubo con una expansión repentina, en el que en uno de sus extremos se colocó un pistón que genera un flujo periódico. Usaron técnicas de visualización y ALD para obtener las componentes axial y radial de la velocidad como función de la posición, en la fase de aspiración del pistón. Enotiadis el al. (1990) estudian la variación cíclica en el flujo haciendo mediciones con ALD en algunos puntos del múltiple de un motor arrastrado. Usan tres métodos diferentes de análisis de los datos y llegan a la conclusión de que el más adecuado es el de promediado en fase.

Shaw et al. (2001) estudian el flujo de aire en estado permanente en un modelo del múltiple de admisión que consta de un plenum de sección rectangular con tres derivaciones o corredores de sección circular. Usando ALD obtienen las dos componentes de la velocidad promedio temporal y la intensidad de turbulencia, para varios puntos sobre el plano central del plenum, encontrando zonas de recirculación cerca de la unión de cada corredor con el plenun.

La dinámica de fluidos computacional abre nuevas alternativas en el estudio de los flujos complejos. En el caso concreto de lo sistemas de admisión se tiene el trabajo de Kumar y Ganesan (2004) quienes obtienen el campo de flujo tridimensional en la admisión de un motor de encendido por chispa para condiciones de estrangulamiento total y parcial usando un código comercial. Señalan lo ventajoso que es para el diseñador el contar con una herramienta computacional que le permita entender el efecto en el flujo de cada uno de los componentes del sistema, lo que facilita su optimización. Validan sus resultados numéricos mediante la comparación de un sólo parámetro global, el flujo másico calculado, con el obtenido experimentalmente.

El presente trabajo tiene como objetivo estudiar mediante anemometría láser Doppler el flujo no permanente en un modelo simplificado del múltiple de admisión muy similar al de Shaw et al. (2001). Se investiga la forma en que evolucionan en el tiempo, durante el 
ciclo de aspiración del motor, las estructuras de flujo que fueron obtenidas por Shaw et al. (2001) para el caso permanente. Los resultados obtenidos son novedosos en si y pueden servir para validar los calculados con algún código numérico, mediante la comparación de los campos de flujo completos.

\section{DESCRIPCIÓN DEL EXPERIMENTO}

El modelo del múltiple de admisión (plenumcorredores) sujeto a estudio, tiene la forma y las dimensiones que se muestran en la Fig. 1.

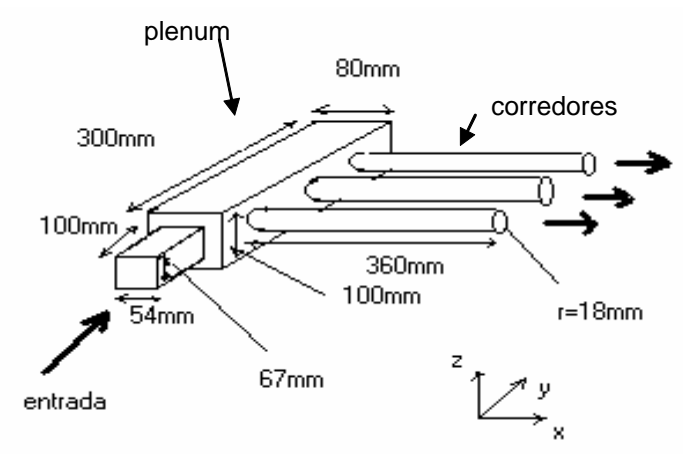

Fig. 1: Modelo propuesto

En los experimentos los corredores se acoplan uno a la vez a la admisión de uno de los cilindros de un motor de combustión interna arrastrado, es decir, que opera sin combustión y accionado mediante un motor eléctrico. Los tres cilindros restantes se dejan abiertos a la atmósfera. El régimen de giro del motor se mantiene constante a $725 \mathrm{rpm}$ y su cilindrada unitaria es de $400 \mathrm{cc}$. Con la finalidad de contar con un punto de referencia en el ciclo del motor, en este caso el punto muerto superior del tiempo de compresión (PMSC), frente a la polea del motor se coloca un sensor óptico reflectivo que responde con pulsos eléctricos cuando la luz infrarroja que emite se refleja en un disco que se coloca en la polea. El disco reflejante contiene una pequeña sección opaca de 2 grados que interrumpe momentáneamente la adquisición de los datos, lo que indica la posición del PMSC.

La velocidad se obtiene mediante un anemómetro láser Doppler de He-Ne de $35 \mathrm{~mW}$, que cuenta con una celda de Bragg que permite determinar el sentido del flujo. Para la obtención de dos componentes de la velocidad, la óptica del anemómetro se gira $90^{\circ}$. El anemómetro completo se monta sobre un sistema de posicionamiento tridimensional independiente que permite ubicar el punto de cruce de los rayos (volumen de medición) en la posición deseada dentro del plenum, que se mantiene fijo. La Figura 2 muestra un esquema del experimento.

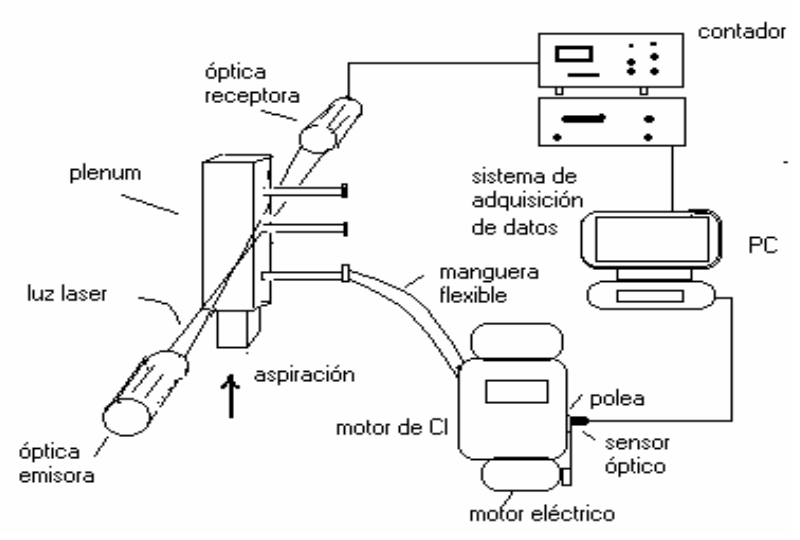

Fig. 2: Arreglo experimental

\section{RESULTADOS}

Para el modelo del múltiple de admisión que se presenta en este trabajo se obtuvieron dos componentes de la velocidad en el plano central del plenum, es decir, el plano que contiene a los ejes de los corredores. La componente de la velocidad perpendicular a dicho plano es relativamente pequeña, lo que concuerda con los trabajos de Durst et al. (1989) y Enotiadis, et al. (1990). Se usa un promediado en fase de las velocidades, tomando 25 ciclos del motor, y se presentan en función del ángulo de giro del cigüeñal, normalizadas con la velocidad media del pistón, que es de 1.98 m/s. La Figura 3 muestra la malla que se usó para hacer las mediciones. Se consideraron tres puntos en la zona de entrada y 130 puntos en el interior del plenum. Nótese que el modelo aparece deformado ya que se tomó una escala diferente en los dos ejes coordenados para ilustrar de una mejor manera la ubicación de los puntos de medición.

\section{Variación temporal de la velocidad}

A la entrada del plenum, en los puntos de coordenadas $(4,1),(4,4)$ y $(4,8)$, el flujo resulta unidireccional, lo que confirma las conclusiones de Arcoumanis y Flamang (1989) y de Shaw et al. (2001). En el cuerpo del plenum se hacen mediciones de las dos componentes de velocidad en cada nodo de la malla, para cada uno de los corredores conectado a la aspiración de manera independiente. Con esto se generan 780 gráficas para el ciclo completo del motor. Aquí se presentan, 
como ejemplo, sólo dos gráficas, que corresponden al punto de coordenadas (7.2, 15) ubicado cerca de la entrada al corredor $A$, y con este corredor conectado al motor. En la Figura 4 se muestra la componente en $x$ de la velocidad como función del ángulo de giro, y en la Figura 5 la componente en y.

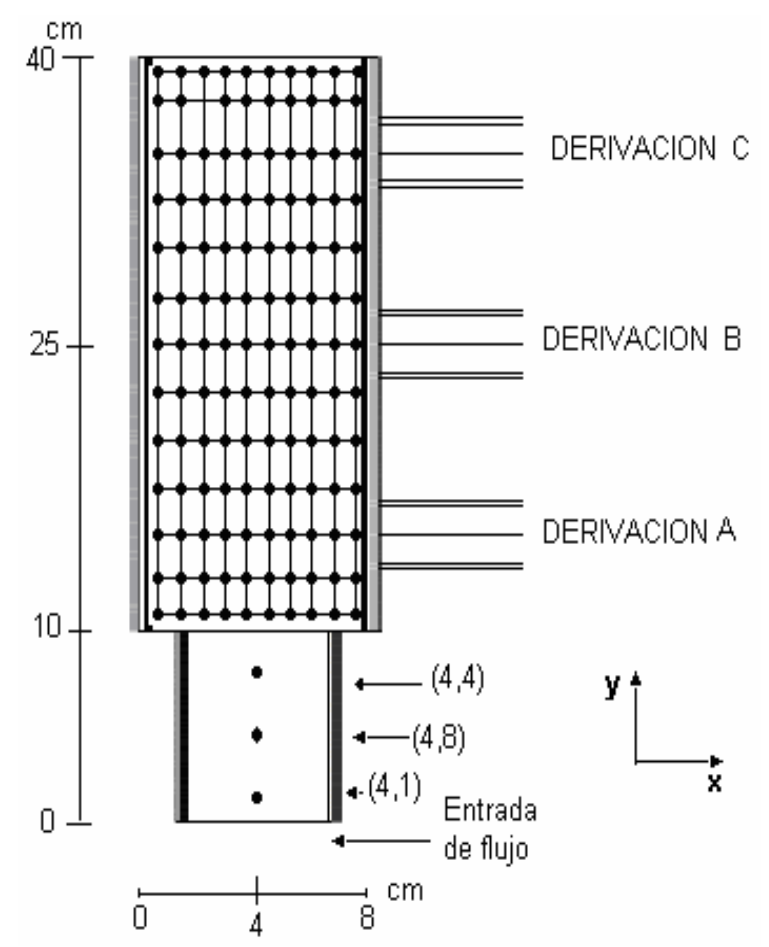

Fig. 3: Puntos de medición en el plano central del modelo

Las dos componentes de la velocidad muestran el reflujo cerca del punto muerto superior y los picos característicos de la reflexión de la onda de presión en los extremos del conducto.

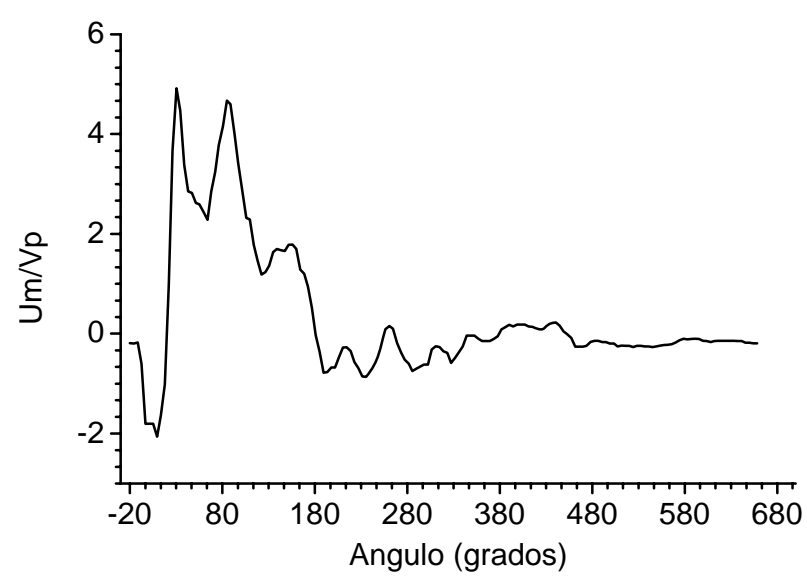

Fig. 4: Componente en $x$ de la velocidad en el punto $(7.2,15)$, con la derivación A operando.

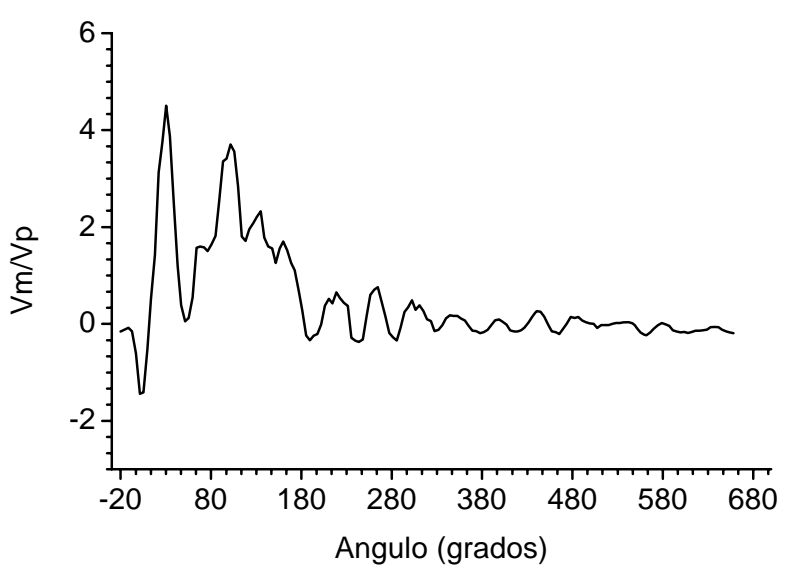

Fig. 5: Componente en y de la velocidad en el punto $(7.2,15)$, con la derivación A operando.

\section{Campos de flujo}

Para tener una idea global del flujo en el plenum se obtienen las velocidades en todos los puntos de la malla para cada 4.23 grados de giro del cigüeñal, lo que permite llevar a cabo animaciones del flujo no estacionario durante todo el ciclo del motor, y a partir de ahí el análisis de sus principales características tales como el reflujo al inicio del proceso de admisión y las zonas de recirculación, entre otras. Desafortunadamente, ya que se tiene en esencia un proceso dinámico, los detalles finos del flujo sólo se aprecian en las animaciones, lo que hace imposible su documentación completa en un artículo como este.

Por razones de espacio, y a manera de ejemplo, en este trabajo sólo se incluirán los campos de flujos correspondientes a dos ángulos de giro (9.3 y 30.2 grados después del punto muerto superior de compresión), para cada uno de los corredores conectado a la aspiración del motor.

En las Figuras 6, 7 y 8 se muestran los campos de flujo para un ángulo de giro de 9.3 grados, con las derivaciones A, B y C conectadas una a la vez al motor. Dado que la válvula de admisión se abre 20 grados antes del PMSC, existe un reflujo del cilindro al múltiple que se mantiene hasta cerca de los 20 grados después del PMSC, y que se manifiesta claramente para este ángulo de 9.3 grados, que es para el que el reflujo alcanza su mayor intensidad. Cuando las derivaciones A y $B$ se encuentran en operación, se presenta una zona de recirculación en la esquina inferior derecha. Cuando se conecta la 
derivación $\mathrm{C}$, la recirculación continúa en esa esquina, pero se presenta otra con más intensidad en la esquina superior derecha. A la salida de las derivaciones también se observa una ligera recirculación.

Las Figuras 9, 10 y 11 muestran el flujo a los 30.2 grados después del PMSC para las tres derivaciones. Para este ángulo el flujo, que ahora se dirige hacia las derivaciones, alcanza una velocidad máxima dentro del ciclo en la mayoría de los puntos. Se observa que aparece una zona de recirculación en la esquina superior izquierda cuando se conecta la derivación $\mathrm{C}$.

\section{DISCUSIÓN}

Como se señaló con anterioridad, resulta difícil documentar con un número limitado de figuras los aspectos dinámicos del flujo, por lo tanto se tendrán que mencionar en este artículo algunos detalles importantes que sólo se pueden observar en las animaciones.

En general se puede decir que la expansión del flujo al pasar del canal de entrada al cuerpo del plenum genera recirculaciones en las esquinas inferior izquierda y derecha, predominando en esta última. Como ya se mencionó, en el canal de entrada el flujo es básicamente unidireccional.

Alrededor de los 60 grados se observa una disminución en la magnitud de las velocidades en todo el campo de flujo, debido a la influencia en el plenum de la onda de rarefacción que se genera en la válvula al inicio del proceso de admisión.

En la región cercana a la entrada de las derivaciones se generan zonas de recirculación, tanto si los conductos descargan al plenum (reflujo), como si el flujo es del plenum a las derivaciones (aspiración normal del motor).

Debido a que en el modelo se tienen esquinas y entradas agudas, se generan zonas de recirculación que disipan parte de la energía que el flujo debiera aprovechar para impulsar el aire al cilindro. En una geometría óptima, además de dar una longitud adecuada a los corredores para aprovechar los efectos de la onda de rarefacción, se debe buscar eliminar estas recirculaciones para incrementar la eficiencia volumétrica.

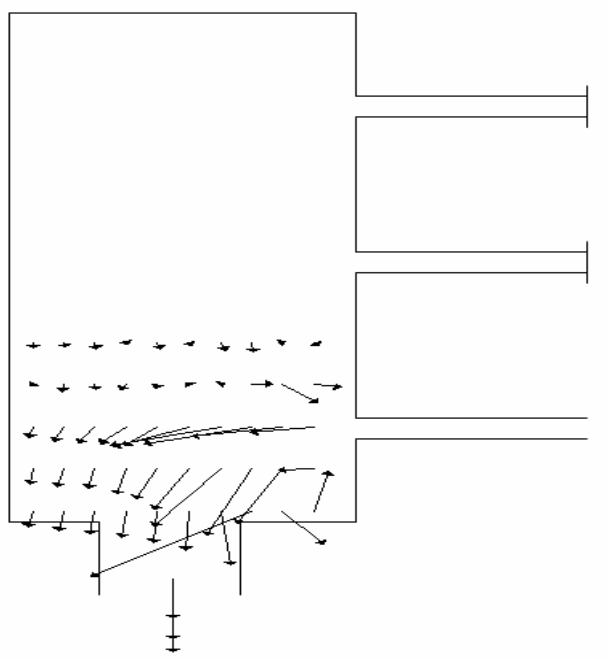

Fig. 6: Campo de velocidades para 9.3 grados, con la derivación A conectada al motor.

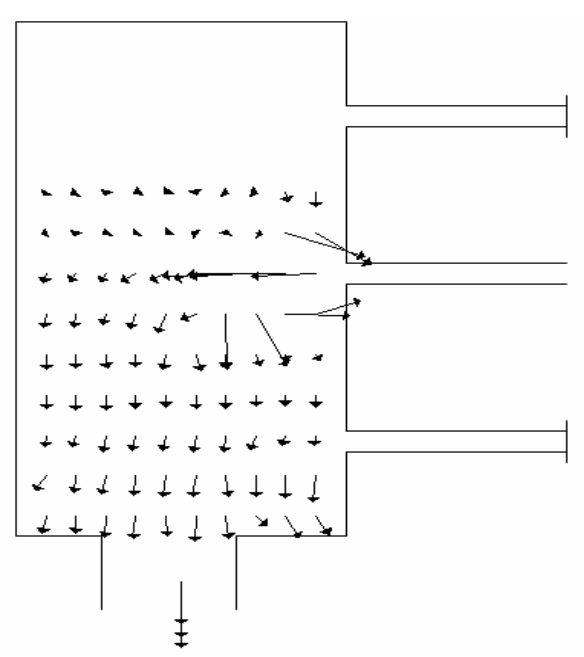

Fig. 7: Campo de velocidades para 9.3 grados, con la derivación B conectada al motor.

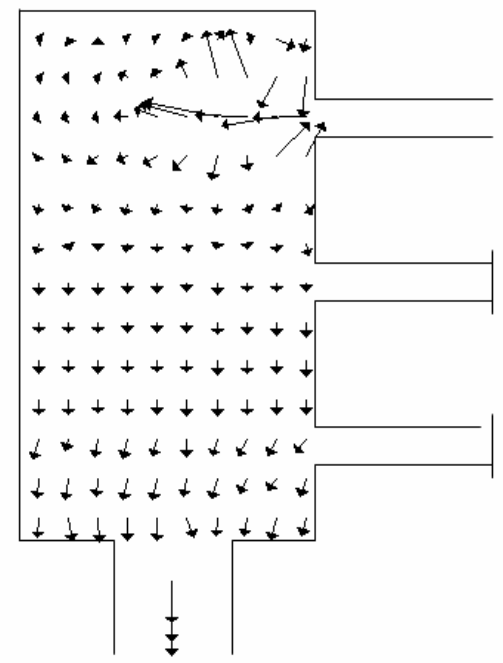

Fig. 8: Campo de velocidades para 9.3 grados, con la derivación $\mathrm{C}$ conectada al motor. 


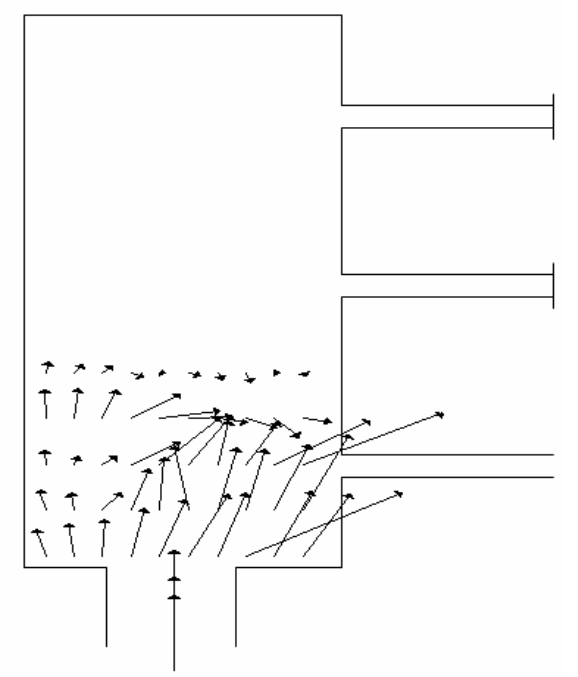

Fig. 9: Campo de velocidades para 30.2 grados, con la derivación A conectada al motor.

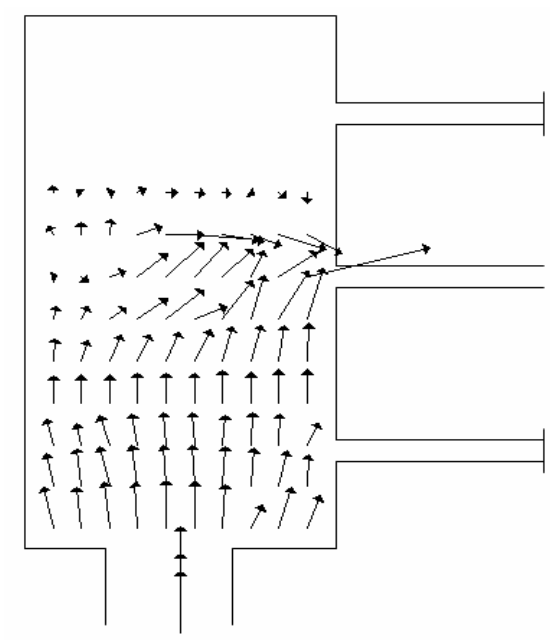

Fig. 10: Campo de velocidades para 30.2 grados, con la derivación B conectada al motor.

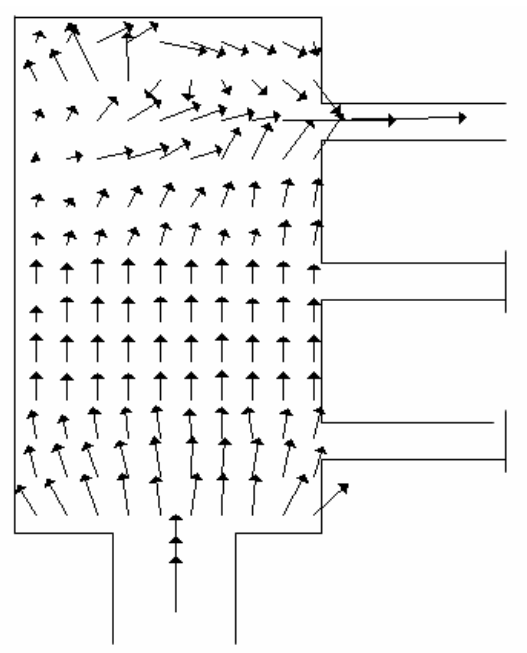

Fig. 11: Campo de velocidades para 30.2 grados, con la derivación $\mathrm{C}$ conectada al motor.

\section{CONCLUSIONES}

El flujo no estacionario en el múltiple de admisión de un motor de combustión interna es bastante complejo. Sin embargo, el uso de modelos simplificados como el presentado en este trabajo, reproduce muchas de las características esenciales de las geometrías reales.

El análisis de las gráficas de la variación temporal de la velocidad para algunos puntos, y la animación del campo de flujo completo, permiten identificar el reflujo que genera el movimiento ascendente del pistón, las zonas de recirculación en las esquinas del plenum y cerca de las entradas a los corredores, así como la influencia de la onda de rarefacción generada al inicio de la admisión y sus reflexiones.

Los resultados experimentales de la presente investigación se pueden usar como condiciones de frontera o iniciales, o en la validación de códigos numéricos sofisticados. También pueden servir como una orientación en el diseño de un múltiple, cundo se trata de evitar la presencia de zonas de recirculación.

\section{REFERENCIAS}

Arcoumanis, C. y Flamang, P., Flow in the inlet manifold of a production diesel engine, Proc. Instn. Mech. Engrs.: 203 (C), 39-49 (1989).

Durst, F., Maxworthy, T., y Pereira, J.C.F., Piston-driven, unsteady separation at a sudden expansion in a tube: Flow visualization and LDA measurement, Phys. Fluids, A: 1 (7), 1249-1260 (1989).

Durst, F., Fukui, K. y Pereira, J.C.F., Fluids dynamics caused cycle- to -cycle variations in piston-driven pipe expansion flows, Experiments in Fluids: 13, 1-10 (1992).

Enotiadis, A.C., Vafidis, C. y Whitelaw, J.H., Interpretation of cyclic flow variations in motored internal combustion engines, Experiments in Fluids: 10, 77-86 (1990).

Kumar, J.S., Ganesan, V., Flow through SI engine air intake system using CFD at part throttle and full throttle, Indian Journal of Engineering and Materials Sciences: 11 (2), 93-99 (2004).

Información Tecnológica - Vol. 18 No 2 - 2007 
Shaw, C.T., Lee, D. J., Richardson, S.H. y Pierson, S., Measuring the flow through a model engine inlet system containing a plenum and runners, Proc. Instn. Mech. Engrs.: 215 (C), 955-964 (2001).
Yeoman, M.L. y Taylor, A., Velocity measurements in the manifold of an internal combustion engine, Laser Velocimetry and Particle Sizing, Doyle Thompson and Warren Stevenson Eds., Hemisphere Publishing Co., 261-266 (1978). 